\title{
ESTABLISHING THE CARDIOTHORACIC RATIO USING CHEST RA- DIOGRAPHS IN AN INDIGENOUS GHANAIAN POPULATION: A SIM- PLE TOOL FOR CARDIOMEGALY SCREENING
}

\author{
Y. B. MENSAH ${ }^{1}$, K. MENSAH ${ }^{2}$, S. ASIAMAH ${ }^{1}$, H. GBADAMOSI ${ }^{1}$, E. A. IDUN ${ }^{3}$, W. $^{2}$ \\ BRAKOHIAPA $^{1}$ and A. ODDOYE ${ }^{4}$ \\ ${ }^{1}$ Department of Radiology, Korle Bu Teaching Hospital, Korle Bu Accra, ${ }^{2}$ Public Health Consultant, P. O. \\ Box GP 15533, Kumasi, ${ }^{3}$ Department of Radiology, 37 Military Hospital, Accra, ${ }^{4}$ Akai House Clinic, Can- \\ tonments, Accra
}

DOI: http://dx.doi.org/10.4314/gmj.v49i3.6

Corresponding Author: Dr. Y.B. Mensah

Email:ybmensah@yahoo.com

Conflict of Interest: None declared

\section{SUMMARY}

Background: Cardiothoracic ratio is a simple and cheap tool in the estimation of heart size. It is a useful index of cardiac size evaluation, and a value of $50 \%$ is generally considered to indicate the upper limit of normal.

Study Objective: This study is to ascertain the normal mean value in cardiothoracic ratio of Ghanaians using chest radiography to serve as baseline for screening for cardiomegaly.

Methodology: Standard postero-anterior radiographs of the -clients/patients were used in the study. The cardiothoracic ratio (CTR) was obtained by dividing the transverse cardiac diameter [sum of the horizontal distances from the right and left lateral-most margins of the heart to the midline (spinous processes of the vertebral bodies)] by the maximum internal thoracic diameter. Systematic sampling with appropriate inclusion and exclusion criteria were used to obtain a sample size of 1989.

Results: The mean transverse cardiac diameter and cardiothoracic ratio increased with age. The transverse thoracic diameter increased with age until the sixth decade when it reduced with age.

The mean CTR increased gradually with age with females having greater values than males. The mean CTR of the study population were $0.459,0.467$ and 0.452 for the general population, females and males respectively.

Conclusion: This study has been able to establish 0.459 as the mean CTR values for Ghanaians. It has also shown the relationship between age and clients /patient's cardiothoracic ratio which compares favourably with findings of a similar study in Nigeria, a neighbouring country in the West African sub region with similar ethnic and social structure.

Keywords: Radiography, Indigenous Ghanaian, cardiomegaly, cardiothoracic ratio, Screening

\section{INTRODUCTION}

The ability to distinguish between normal and abnormal is fundamental to the practice of medicine. In the field of chest radiology much observation and interpretation is necessarily pictorial and judgmental. ${ }^{1}$ Assessing heart size has been and remains an important and useful diagnostic parameter in chest radiograph evaluation. ${ }^{2}$

Chest radiographs permit both a subjective appraisal of heart size as well as measurements of the cardiac silhouette which may, in turn, be related to the size of the thorax. ${ }^{2,3}$ Studies have shown a satisfactory correlation (greater than 0.7) between the heart size measured on radiographs and that found at postmortem. ${ }^{4}$

Chest radiography is the most commonly used modality for the detection of cardiomegaly and the cardiothoracic ratio has been used as an important indicator of cardiac size. ${ }^{5}$ It is a simple and cheap tool used in the estimation of heart size ${ }^{6}$ and a value of $50 \%$ is generally considered to indicate the upper limit of normal. However, this value is not always correct and many cardiac and extracardiac factors influence this measure, such as the examination technique, the patient's biotype, the patient's physiological status, thoracic alterations (scoliosis, pectus excavatum), and the size of the lungs, the breathing phase, the cardiac cycle phase, and heart rate at the time of examination. ${ }^{2,3,7}$

The cardiothoracic ratio is derived from two measurements:

i. The widest internal diameter of the thoracic cavity from the medial border of the ribs i.e. pleura to pleura and

ii. The transverse diameter of the heart which is obtained as the sum of the horizontal distance between the left and right lateral most borders 
of the heart and the midline (spinous processes of the vertebral bodies).

The cardiothoracic ratio is therefore expressed as the ratio of the transverse diameter of the heart to the maximum internal diameter of the thoracic cavity. ${ }^{1,8}$ Determination of the heart size including the size of each of the four chambers of the heart is a very important measurement which helps clinicians ascertain the aetiology of cardiomegaly.

Though heart chamber size can be determined by chest radiography, the diagnosis can be made more accurately by other modalities like cardiac echocardiography, magnetic resonance imaging (MRI) and computed tomography (CT scan). ${ }^{9},{ }^{10}$ Echocardiography is commonly superior to chest radiography in providing a better assessment of heart chamber size and function. It has been shown to have a superior sensitivity and specificity in determining cardiac chamber size, however its cost and availability makes it impractical and expensive to obtain an echocardiogram on every patient. ${ }^{9} \mathrm{CT}$ scan and MRI machines like echocardiography can give accurate assessment of cardiac chamber size and function.

They can also be used to assess the mediastinum, the thoracic cage, coronary vessels. CT scan has an added advantage of having a very high sensitivity and specificity in diagnosing lung parenchyma lesions but a major disadvantage of giving patients a very high dose of radiation relative to general radiography.

Chest radiographs are readily available, inexpensive and allow a straightforward and inexpensive assessment of heart size and its changes over time. Currently in Ghana, almost every district hospital has general radiography (x-ray) services while CT scan and MRI machines with the ability to evaluate cardiac chambers and function are only available in Accra and Kumasi (the two largest cities in Ghana). To this end, the use of cardiothoracic ratio as an effort to determine the size of the heart remains the only option available to the majority of clinicians in the country. ${ }^{9}$

Earlier research has revealed ethnic differences in the cardiothoracic ratio of Caucasians, Asians and people of African descent. ${ }^{1,8}$ These studies have provided an upper limit of normal for Caucasians as $50 \%$ and that for people of African descent as 55\%. These figures have been accepted and are being used in most African countries. A similar study done in the Rivers State in Nigeria revealed that the cardiothoracic ratio of the Nigerian $(46.3 \%)$ is slightly larger than that of the Caucasian (46\%) but remains less than $50 \% .{ }^{10}$ No such study has been done to find the cardiothoracic ratio of
Ghanaians. This study is to ascertain the normal mean cardiothoracic ratio of Ghanaians using chest radiographs to serve as baseline for screening for cardiomegaly.

\section{Delimitation of the Study}

The study sought to ascertain an approximate normal range/value for the cardiothoracic ratio assessed on chest radiographs of Ghanaians chosen at random without knowing their weight, height or blood pressure. It did not seek to find any relationship or correlation between cardiothoracic ratio and the height, weight and/or blood pressure of the clients/patients.

\section{METHODS}

The study was a retrospective study conducted at Akai House Clinic, Accra, Ghana from October 2009 to November 2010. Akai House Clinic is a clinic with modern state of the art diagnostic equipment. A total of 1989 chest radiographs of indigenous Ghanaians (Indigenous Ghanaian-a person bearing a typical Ghanaian name signifying he/she has at least one Ghanaian parent) were evaluated during the study period.

The clients/patients were sent by clinicians for chest radiographs and were not re-examined by the researchers. The research used the clinician's diagnosis to disqualify patients with cardiac or chest problems as well as patients whose subsequent chest radiograph showed cardiac or thoracic cage abnormality. This was done to reduce the probability of using radiographs of clients/patients with cardiac problems and/or abnormalities.

An average of 40 chest radiographs were taken at the study centre each day which works to a total of 11,400 chest radiographs as the study population over the study period. Some of these radiographs were existing radiographs and others were taken during the study period. Existing radiographs used bore a typical Ghanaian name, while clients/patients whose radiographs were taken during the study period were asked of their Ghanaian nationality before being included. Sampling was done using systematic sampling.

Most previous studies had used samples of around 1500 patients so it was decided to use a sample of more than 1500 patients to improve the accuracy. Using 1500 patients as in previous studies would have given a sampling interval of 7 so it was decided to use a sampling interval of 3 so that in the event of some existing radiographs/new radiographs not meeting the inclusion criteria, there will still be more than 1500 radiographs selected for the study. 
Radiographs used were for clients/patients who took the chest radiograph as part of a medical check-up for employment, school or visa application. This was partly to ensure that the patient did not present with an illness (whether cardiac illness or not) but this was not taken in isolation but with the exclusion criteria.

\section{Exclusion criteria:}

1. Radiographs of clients/patients with thoracic cage abnormalities, obvious cardiac enlargement (on visual inspection of the radiograph).

2. Radiographs of clients/patients with abnormal heart shape and/or evidence of significant rotation.

3. Radiographs of clients/patients whose request form indicated any respiratory or cardiac disorder.

The sample obtained came out as 3,800 patients and after eliminating those that did not meet the inclusion criteria, which fell out because of the exclusion criteria, the final sample was 1989 radiographs. The sample of 1989 radiographs in a population of 11400 gave a confidence interval of 2.42 at the $95 \%$ confidence level and calculating the sample size from this gave a sample size of 1434 at the $95 \%$ confidence level, thus the sample of 1989 used would decrease the margin of error.

Chest radiographs were taken using a digital radiography machine, GE Brivo DR-F. Standard posteroanterior radiographs were taken with the patient upright, at maximum inspiration and with a tube-detector distance of $180 \mathrm{~cm}$.

Those whose request form indicated any respiratory or cardiac disorder were also excluded from the study. The transverse cardiac diameter (TCD) was obtained by summing the horizontal distances from the lateral most margins (right and left) of the heart borders to the midline (spinous processes of the vertebral bodies) Figure 1.

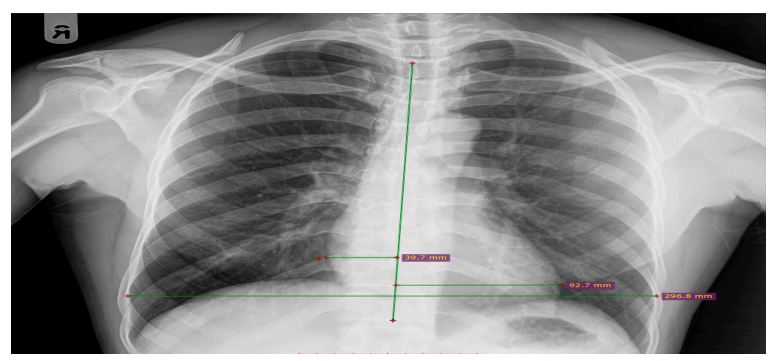

Figure 1 A chest radiograph (PA) showing how cardiothoracic ratio is measured

The widest transverse thoracic diameter (TTD) was obtained by measuring the widest distance between the medial aspects of the ribs i.e. pleura to pleura.
The cardiothoracic ratio (CTR) was obtained by dividing the transverse cardiac diameter by the maximum internal thoracic diameter. Data obtained was summarized and analyzed using Microsoft Excel 2010.

\section{RESULTS}

A total of 1989 radiographs of indigenous Ghanaians were evaluated for the study out of which $998(50.2 \%)$ were that of males and 991(49.8\%) were that of females. The mean ages of the study population were 30.9 years (Standard Deviation (SD) -12.4), 31.2 years (SD- 13.0) and 30.6 years (SD- 11.8) for the general population, females and males respectively. The mean TCD increased gradually with age, with males having slightly greater diameters than females as shown on Figure 2. The mean TCD of the study population were $126.1 \mathrm{~mm}$ (SD- 14.1; Margin of Error for 95\% Confidence Interval (ME)- 0.6), 122.1mm (SD-13.1, ME0.8 ) and $129.8 \mathrm{~mm}$ (SD-13.9, ME- 0.9) for the general population, females and males respectively.

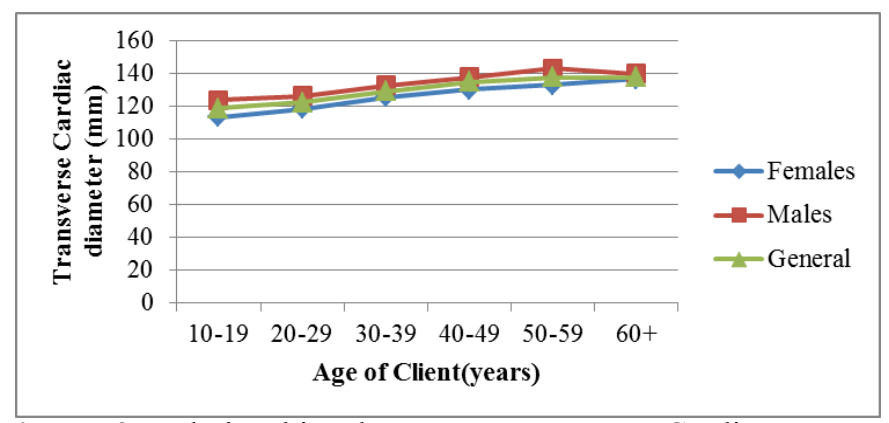

Figure 2 Relationships between Transverse Cardiac Diameter and Age

The study population was further divided into age groups and the mean CTR of each of these groups using a 95\% confidence interval was obtained as shown in Table 1. The mean CTR of clients/patients (general) less than 60 years was less than $50 \%$, while that for the elderly was above $50 \%$. The mean CTR for males of all the age groups and females less than 60 years were less than $50 \%$ while that for females aged 60 years or more was $52.4+4.8 \%$.

The mean TTD of the study population were $276.0 \mathrm{~mm}$ (SD, 58.1; Margin of Error for 95\% Confidence Interval (ME), 2.6), 263.7mm (SD, 78.9, ME, 4.9) and $287.7 \mathrm{~mm}$ (SD, 19.3, ME, 1.2) for the general population, females and males respectively. 
Table 1 Cardiothoracic Ratios of Various Age-Groups

\begin{tabular}{|c|c|c|c|c|c|c|c|}
\hline $\begin{array}{l}\text { Catego- } \\
\text { ry }\end{array}$ & Age-Group & $\begin{array}{l}\text { Popula- } \\
\text { tion }\end{array}$ & $\begin{array}{l}\text { Mean } \\
\text { CTR }\end{array}$ & SD & ME & CI & CI (\%) \\
\hline \multirow{7}{*}{ General } & $10-19$ & 330 & 0.447 & 0.035 & 0.0037 & $0.447 \pm 0.004$ & $44.7 \pm 0.4$ \\
\hline & $20-29$ & 722 & 0.449 & 0.042 & 0.0030 & $0.449 \pm 0.003$ & $44.9+0.3$ \\
\hline & $30-39$ & 551 & 0.460 & 0.038 & 0.0031 & $0.460 \pm 0.003$ & $44.7 \pm 0.3$ \\
\hline & 40-49 & 209 & 0.474 & 0.042 & 0.0056 & $0.474 \pm 0.006$ & $47.4+0.6$ \\
\hline & $50-59$ & 90 & 0.492 & 0.045 & 0.0093 & $0.492 \pm 0.009$ & $49.2+0.9$ \\
\hline & $60+$ & 87 & 0.509 & 0.050 & 0.0105 & $0.509 \pm 0.011$ & $50.9+1.1$ \\
\hline & Total & 1989 & 0.459 & 0.044 & 0.0019 & $0.459 \pm 0.002$ & $45.9+0.2$ \\
\hline \multirow{7}{*}{ Female } & $10-19$ & 159 & 0.448 & 0.038 & 0.0058 & $0.448 \pm 0.006$ & $44.8+0.6$ \\
\hline & $20-29$ & 357 & 0.456 & 0.043 & 0.0044 & $0.456 \pm 0.004$ & $45.6 \pm 0.4$ \\
\hline & 30-39 & 260 & 0.470 & 0.038 & 0.0045 & $0.470 \pm 0.005$ & $47.0+0.5$ \\
\hline & $40-49$ & 114 & 0.484 & 0.040 & 0.0073 & $0.485 \pm 0.007$ & $48.5 \pm 0.7$ \\
\hline & $50-59$ & 44 & 0.491 & 0.043 & 0.0127 & $0.492 \pm 0.013$ & $49.2+1.3$ \\
\hline & $60+$ & 57 & 0.523 & 0.048 & 0.0123 & $0.524 \pm 0.048$ & $52.4+4.8$ \\
\hline & Total & 991 & 0.467 & 0.045 & 0.0028 & $0.467+0.003$ & $46.7+0.3$ \\
\hline \multirow{7}{*}{ Male } & 10-19 & 151 & 0.447 & 0.032 & 0.0051 & $0.447 \pm 0.005$ & $44.7 \pm 0.5$ \\
\hline & 20-29 & 365 & 0.443 & 0.039 & 0.0040 & $0.443+0.004$ & $44.3+0.4$ \\
\hline & 30-39 & 291 & 0.452 & 0.035 & 0.0040 & $0.452 \pm 0.004$ & $45.2+0.4$ \\
\hline & 40-49 & 115 & 0.466 & 0.041 & 0.0075 & $0.466 \pm 0.008$ & $46.6 \pm 0.8$ \\
\hline & 50-59 & 46 & 0.483 & 0.046 & 0.0132 & $0.483 \pm 0.013$ & $48.3+1.3$ \\
\hline & $60+$ & 30 & 0.481 & 0.004 & 0.0015 & $0.481 \pm 0.002$ & $48.1+0.2$ \\
\hline & Total & 998 & 0.452 & 0.039 & 0.0024 & $0.452+0.002$ & $45.2+0.2$ \\
\hline
\end{tabular}

The mean TTD increased gradually with age till the fifth decade and then reduced after that. Males have greater diameters than females as shown in Figure 3.

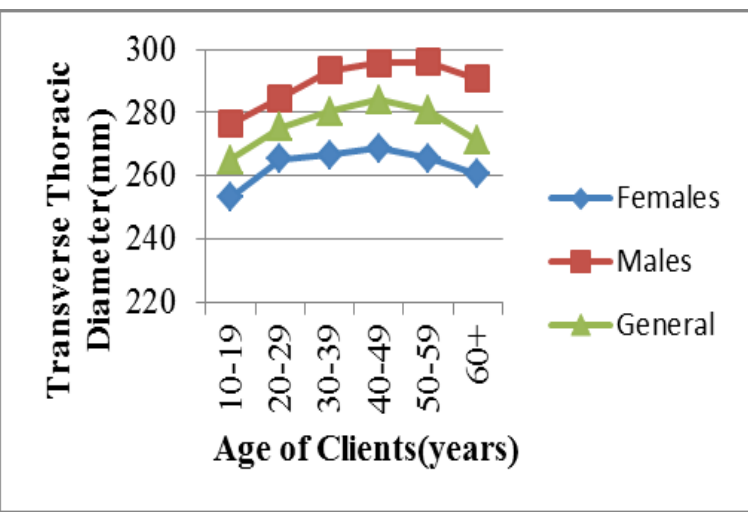

Figure 3 Relationships between Transverse Thoracic Diameter and Age

The mean CTR increased gradually with age with females having greater values than males as shown on Figure 4.
The mean CTR of the study population were 0.459 (SD- 0.044; Margin of Error for 95\% Confidence Interval (ME)- 0.002), 0.467 (SD-0.045, ME- 0.003) and 0.452 (SD-0.39, ME- 0.002) for the general population, females and males respectively.

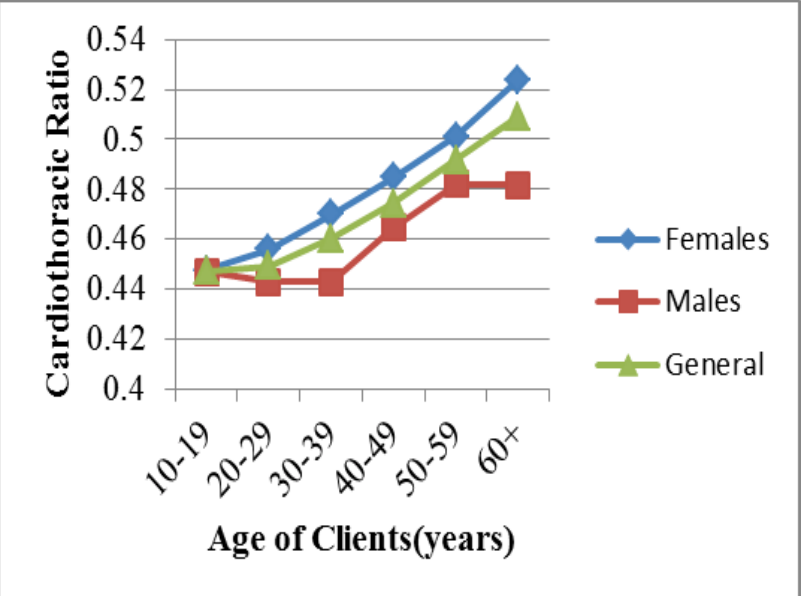

Figure 4 Relationships between Cardiothoracic Ratio and Age 


\section{Limitations of the Study}

Radiographs of asymptomatic clients/patients were used in the study. However absence of symptoms does not necessarily mean absence of disease.

\section{DISCUSSION}

Cardiothoracic ratio is a very simple and useful tool which can serve as an index of cardiac size in screening for cardiovascular diseases. ${ }^{6}$ When the normal value for the local population is known, it serves as a baseline for proper assessment.

Our study found that transverse cardiac diameter (TCD) increased gradually with age. This trend was also noted in other studies that have been done on Caucasians, Asians and people of African descent. This increase in transverse cardiac diameter with age has been attributed to increased cardiac ventricular muscle thickness resulting from increased vascular resistance or loss of elasticity of the great vessels. ${ }^{8}$

This study like the other studies mentioned in the paragraph above $\mathrm{e}^{1,8,11-13}$ noted an increase in the transverse thoracic diameter (TTD) with age till the sixth decade after which it reduced. The reduction in TTD has been attributed to reduced ribcage mobility and compliance in the elderly. ${ }^{8}$

The cardiothoracic ratio (CTR) was also noted to increase gradually with age in this study, a finding which has been documented in the other studies discussed earlier in this chapter. ${ }^{1,8,11-13}$ The gradual increase in CTR up to age 50 years is believed to be due to gradual increase in TCD. The reduction in the TTD also comes into play in the elderly leading to additional increase in CTR. The factors responsible for these changes have already been discussed earlier in this chapter.

The mean CTR of the study population were $45.9 \%$ $+0.2,46.7 \%+0.3$ and $45.2 \%+0.2$ for the general population, females and males respectively. This result compared favourably with a similar study in Nigeria where the mean CTR values were $45.7 \% \pm 0.03$ for males, $46.7 \% \pm 0.04$ for females and $46.3 \% \pm 0.03$ for both males and females. ${ }^{9}$

The 95\% confidence interval for the mean CTR for all males and females less than 60 years was found to be less than $50 \%$ (shown in fig 3 and table 1 ). This trend is similar to what has been noted in other studies. $1,8,12,13$ Elderly females on the other hand had a figure greater than $50 \%(52.4+1.2 \%)$. A similar trend was noted in a longitudinal study on changes of CTR in women which showed that about $20 \%$ of elderly women will have a CTR greater than $50 \%{ }^{8}$
This was attributed to the fact that the reduction in TTD with age was more pronounced in elderly women. ${ }^{8}$ However the situation was slightly different in the study in the Rivers state in Nigeria where mean CTR for all age-groups was less than $50 \%{ }^{10}$ This might possibly be due to the average Nigerian being taller and bigger than the average Ghanaian, though there is no empirical evidence for this.

A study done to evaluate the changes of CTR with age in men (aged 23-76 years) showed that only 3\% had a CTR greater than $50 \%$. This finding is similar what was found in our study. ${ }^{13}$ Various studies have shown that though the cardiothoracic ratio is a very useful basic tool for the evaluation of heart size, it fares poorly when it comes to evaluation of both the size of the heart and the sizes of its chambers. ${ }^{9,14}$

A study, which compared the sensitivity and specificity of cardiothoracic ratio, noted that cardiothoracic ratio had a fairly high sensitivity (85 to $90 \%$ ) but low specificity (14 to $17 \%$ ) when used to determine whether the heart is enlarged or not. Echocardiography is therefore the modality to go for when a more objective numerical estimation of the cardiac size (and the sizes of its chambers) is needed because of its high sensitivity and specificity.

The study recommends that because it is easy and cheap to measure the CTR by the chest X-ray for any patient, it can be regarded as a preliminary measure to the size of the heart, especially in the numerous settings where X-ray remains the only available diagnostic facility but the echocardiographic measurement remains the more accurate. ${ }^{9}$

\section{CONCLUSION}

This study has been able to establish 0.459 as the mean CTR values for Ghanaians. It has also shown the relationship between age and client's cardiothoracic ratio, transverse cardiac diameter and transverse thoracic diameter. These figures compare favourably with findings of a similar study in Nigeria, a neighbouring country in the West African sub region with similar ethnic and social structures like Ghana (as has been shown in table 1). The values will be very valuable in screening Ghanaians for cardiomegaly.

\section{REFERENCES}

1. Nickol K., Wade A.J. Radiographic heart size and cardiothoracic ratio in three ethnic groups: a basis for a simple screening test for cardiac enlargement in men. British Journal of Radiology,1982; 55: 399-403 
2. Nakamori N, Doi $\mathrm{K}$, MacMahon $\mathrm{H}$, Yasuo $\mathrm{S}$, Montner S. Effect of heart-size parameters computed from digital chest radiographs on detection of cardiomegaly: potential usefulness for computer-aided diagnosis. Invest Radiol 1991; 26:546550 .

3. Perez A. A, Ribeiro A. L. P, Barros M. V. L, de Sousa M. R, Bittencourt R. J, Machado F. S, Rocha M. O. C. Value of the radiological study of the thorax for diagnosing left ventricular dysfunction in Chagas' disease. Arq. Bras. Cardiol. 2003; 80(2)

4. Murphy M. L, Blue L. R, Thenabadu P. N, Phillips J. R, Ferris E. J. The reliability of the routine chest roentgenogram for determination of heart size based on specific ventricular chamber evaluation at postmortem. Invest Radiol. 1985; 20(1):21-5.

5. Tomaszewski R., Cardiomegaly in Tropical Africa. Int Marit Health 2012; 63, 1: 56-58

6. Justin M, Zaman S, Sanders J, Crook A. M, Feder G, Shipley M, Timmis A, Hemingway H. Cardiothoracic ratio within the "normal", range independently predicts mortality in patients undergoing coronary angiography. Heart 2007;93:491-494.

7. Hada Y. Cardiothoracic ratio. J. Cardiol. 1995 Jul; 26(1):51-4.
8. Inouea K, Yoshiib K, Itob H. Effect of Aging on Cardiothoracic Ratio in Women: A Longitudinal Study. Gerontology 1999;45:53-58

9. Kadhum A. A., Sharif S., Abd-Hazaa M.. Validity of Chest X-Ray In Estimation of Cardiac Size in Comparison To Echocardiography. MJBU. 2007; 25(2): 48-51

10. Ghali J. K., LiaoY., Simmons B., Castaner A., Guichan C., Cooper R.S. The prognostic role of left ventricular hypertrophy in patients with or without coronary artery disease. Ann Intern Med 1992; 117: 831-836.

11. Oladipo G.S., Okoh P.D., Kelly E.I., Arimie C.O.D., Leko B. J.. Normal Heart Sizes of Nigerians Within Rivers State Using Cardiothoracic Ratio. Scientia Africana. 2012 Dec; 11 (2), 9- 21.

12. Lauder I. J, Milne J. S. Longitudinal study of heart size in older people. British Heart Journal, 1976, 38: 1286-1290.

13. Potter J. F, Elahi D, Tobin J. D, Andres R. Effect of aging on the cardiothoracic ratio of men. $\mathrm{J} \mathrm{Am}$ Geriatr Soc. 1982;30(6):404-9.

14. Lupow J.B., Sivak S.L., Boss D.. The Accuracy of the Cardiothoracic Ratio as a Predictor of Cardiac Enlargement and Dysfunction. Acad Emerg Med 2003; 9(5): 462. 